\title{
Myeloid Sarcoma with Megakaryoblastic Differentiation Arising in the Conjunctiva
}

\author{
Frederick A. Jakobiec ${ }^{a}$ Natalie Wolkow ${ }^{a}$ Fouad R. Zakka ${ }^{b}$ Peter A.D. Rubin ${ }^{c}$ \\ aDavid G. Cogan Laboratory of Ophthalmic Pathology, Department of Ophthalmology, Massachusetts Eye and Ear \\ Infirmary, Harvard Medical School, Boston, MA, USA; ${ }^{b}$ Department of Pathology and Laboratory Medicine, Alpert \\ Medical School, Brown University, Providence, RI, USA; ' ${ }^{C}$ Eyelid Experts, Palm Beach Gardens, FL, USA
}

\section{Established Facts}

- Myeloid sarcoma is an extramedullary deposit of malignant myeloblasts that eventually evolves into a rapidly fatal leukemia.

\section{Novel Insights}

- A myeloid sarcomatous proliferation with megakaryoblastic differentiation developed in the conjunctiva and was associated with an aggressive clinical course, trilinear hematopoietic abnormalities of stem cells, evidence of a prodromal myelodysplasia, $\mathrm{t}(12 ; 17)$ translocation and trisomy 8 , the latter worsening the prognosis in elderly patients.

\section{Keywords}

Myeloid sarcoma - Granulocytic sarcoma - Chloroma . Acute megakaryoblastic leukemia · Conjunctiva · Blast . Megakaryocyte - Myelofibrosis - JAK2 - V617F mutation . Trisomy $8 \cdot \mathrm{t}(12 ; 17)$ translocation

\section{Abstract}

An 87-year-old woman not known to have either a lymphoma or leukemia developed a left multinodular, fish-flesh superior epibulbar and forniceal mass. A biopsy disclosed a blastic tumor with scattered multinucleated immature megakaryoblasts. Immunophenotyping of bone marrow cells revealed strong positivity for CD7, CD31, CD43, CD45, CD61, and CD117; CD71, myeloperoxidase, and lysozyme were also positive in scattered cells. Forty percent of the neoplastic cells were Ki-67 positive. Cytogenetic studies indicated a trisomy 8 (associated with worse prognosis) and a t 12 ; 17) translocation. Desmin, smooth muscle actin, pancytokeratin, CAM 5.2, adipophilin, tryptase, S100, SOX10, MART1, and E-cadherin were negative, ruling out a nonhematopoietic tumor. The conjunctival lesion was diagnosed as a myeloid sarcoma with megakaryoblastic differentiation, a rare variant. It probably arose from a myelodysplastic syndrome. This is the first case of its type to develop in the conjunctiva.

(c) 2018 S. Karger AG, Basel

F.R. Zakka: formerly fellow in Eye Pathology, Massachusetts Eye and Ear Infirmary, Harvard Medical School.

\section{KARGER}

(c) 2018 S. Karger AG, Basel

E-Mail karger@karger.com

www.karger.com/oop
Frederick A. Jakobiec, MD, DSc

David G. Cogan Laboratory of Ophthalmic Pathology Department of Ophthalmology, Massachusetts Eye and Ear Infirmary 243 Charles Street Suite 208, Boston, MA 02114 (USA)

E-Mail Fred_Jakobiec@meei.harvard.edu 


\section{Introduction}

Myeloid sarcomas (granulocytic sarcoma or chloroma) are localized extramedullary tumors composed of myelogenous blastic cells that destroy their resident tissue $[1,2]$. Therefore, subclinical infiltration of an organ by leukemic cells without creating a mass does not constitute a myeloid sarcoma, especially if there is no destruction of the local tissue. For example, unblemished and nonnodular skin that microscopically has infiltrating leukemic tumor cells does not qualify as a granulocytic sarcoma. If the sarcoma is extramedullary (proliferation outside of the bone marrow compartment), the main sites of alternative growth are the liver, spleen, lymph nodes, skin, mucous membranes, orbits, central nervous system, bones, gonads, and other viscera. Granulocytic sarcomas may precede, be concurrent with, or follow the emergence of leukemia. In this article, we describe an elderly patient who presented with an unusual conjunctival mass that was ultimately determined to be the first case of conjunctival myeloid sarcoma with megakaryoblastic differentiation. We place the lesion in a detailed clinicopathologic context of ocular myeloid sarcoma and describe its immunophenotypic and cytogenetic characteristics.

\section{Case Report}

An 87-year-old woman presented with left upper eyelid swelling and discomfort, which had been worsening during the course of a year. Six months previously, the lesion had been diagnosed elsewhere as a chalazion. Treatment with warm compresses and topical steroid-antibiotic drops had not improved her condition. Her ocular history was notable only for allergic conjunctivitis and bilateral pseudophakia, and her medical history was remarkable for hypertension, hyperlipidemia, cardiac conduction defects requiring a pacemaker, congestive heart failure, and thrombocytopenia.

Physical examination revealed fullness of the left upper eyelid with entropion and ptosis, which was most prominent nasally (Fig. 1a). A firm, moderately tender mass was palpated within the left upper eyelid. Eversion demonstrated a lobulated subconjunctival mass in the superior epibulbar surface and fornix (Fig. 1b). No regional lymphadenopathy was palpated. Computed tomography with contrast demonstrated a well-demarcated mass confined to the left conjunctiva and anteriormost orbit (Fig. 1c, d). An attempt at an excisional biopsy was performed. Intraoperatively, the left upper eyelid was everted revealing a $3 \times 2 \mathrm{~cm}$ multinodular tumor that was firmly adherent to the superior tarsus and extended into the superior conjunctival fornix (Fig. 1e).

Grossly the specimen measured $3.5 \times 2.5 \times 1 \mathrm{~cm}$. Frozen section evaluation demonstrated that the tumor was present at all inked margins. A portion of the remaining tissue was submitted for flow cytometry and the rest for permanent sections. Histopathologic evaluation of slides sent to the Massachusetts Eye and Ear Infir- mary revealed a tumor composed of round epithelioid-type blastic cells with large nuclei, prominent nucleoli, and a finely granular nucleoplasm (Fig. 2a). Some cells grew in clusters or groups and had abundant cytoplasm with two or more nuclei (Fig. 2b, inset). Thin collagen strands demarcated the clusters (Fig. 2b); they were reticulin (Fig. 2c), trichrome and PAS positive. The tumor was mitotically active (10 mitoses/hpf; approximately $40 \%$ of cells were Ki-67 positive) (Fig. 2d). The tumor cells were strongly positive for CD45 (Fig. 2e), CD7, CD31, CD43 (Fig. 2f), CD61 (Fig. 2g), CD117 (Fig. 2h), FLI-1, and factor VIII, while ERG was positive in approximately $60 \%$ of the tumor cells. Myeloperoxidase and lysozyme showed scattered positivity in the large tumor cells; p53 was strongly positive in approximately $5 \%$ of tumor cells; OCT3/4 showed faint background staining; CD71 showed sparse staining in some tumor cells. CD3 was positive in scattered small T cells; Alcian blue demonstrated mild stromal mucin at the lesion's periphery. The following immunohistochemical and special stains were negative: CD20, CD30, CD34, CD56, CD68, SMA, desmin, S100, SOX10, MART-1, e-cadherin, EBER, EMA, CAM5.2, pankeratin, adipophilin, Giemsa, and tryptase. These findings were in keeping with a hematologic neoplasm, most consistent with myeloid sarcoma (megakaryoblastic type). Flow cytometry of the conjunctival tumor tissue showed immunophenotypic evidence of an abnormal cell population in approximately $94.3 \%$ of all analyzed events. This result was found in the dim to negative CD45 region/ blast gate that expresses CD7, partial HLA-DR, partial CD33, and partial CD34 in keeping with the diagnosis.

Bone marrow biopsy with karyotyping revealed an increase in blasts of myeloid origin accounting for $8.8 \%$ of total leukocytes. These blasts expressed CD13, CD33, C34, cytoplasmic myeloperoxidase, and aberrant CD7. Fluorescence in situ hybridization (FISH) detected two unrelated abnormal clones, one with a $t(12$; 17) translocation of uncertain significance and the other with trisomy 8 in $4 \%$ of cells, which portends a poor prognosis in elderly patients [3]. The marrow was hypercellular and displayed marked dysplasia of all three cell lines and increased reticulin fibers. Megakaryocytes were increased in number, forming large tight clusters with abnormal cellular morphology. The final impression was that the patient had a myelodysplastic syndrome.

Azacitidine (Vidaza ${ }^{\circledR}$, Celgene, Summit, NJ, USA) injections were initiated but halted after three treatments due to worsening of the patient's anemia (a known medication side effect) that required blood transfusions. Over the subsequent 3 months her condition worsened. Repeat peripheral blood counts demonstrated a white blood cell count greater than $30 \times 10^{9} / \mathrm{L}$, with numerous immature myeloid cells, suggestive of an evolving myelogenous leukemia. In short order, the patient developed pneumonia, worsening congestive heart failure, renal failure, urosepsis and died.

\section{Discussion}

Despite being an uncommon ocular adnexal lesion, there is no ocular adnexal site that has been spared involvement by myeloid sarcoma. With respect to the current case, conjunctival lesions are notably exceptional. Some clinicians consider all eyelid lesions as a single group. Greater precision, however, is required. With care, 


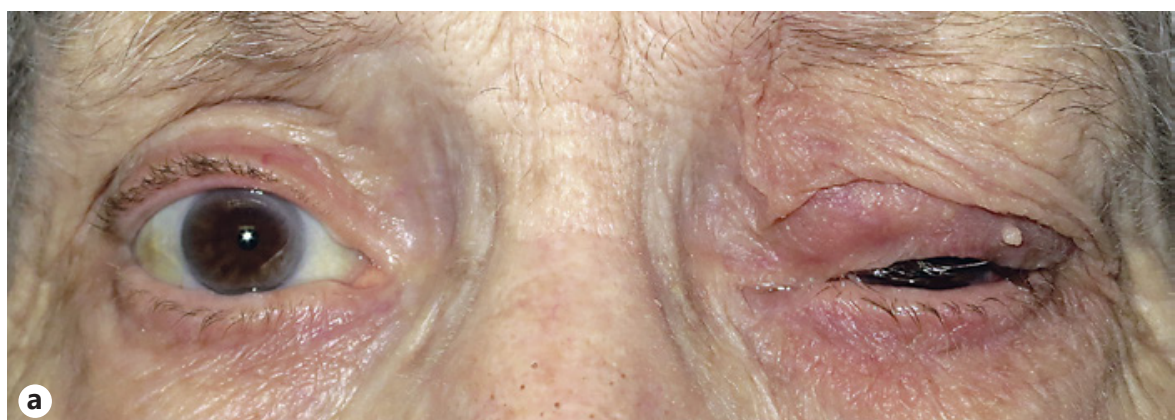

Fig. 1. Clinical features of a myeloid sarcoma with megakaryoblasts. a There is swelling and mild erythema of the skin of the eyelids in an 87-year-old woman who developed upper medial eyelid fullness over the preceding year. b A pale superior forniceal lesion with smaller medial nodules. c Axial CT scan reveals restriction of the mass to the conjunctival sac. $\mathbf{d}$ Sagittal CT scan demonstrates modest trailoff of the lesion into the anterior orbit along the tissue planes surrounding the superior and inferior rectus muscles. e On eversion of the left upper eyelid, a multinodular, fishflesh excrescent, nonulcerated mass is evident.
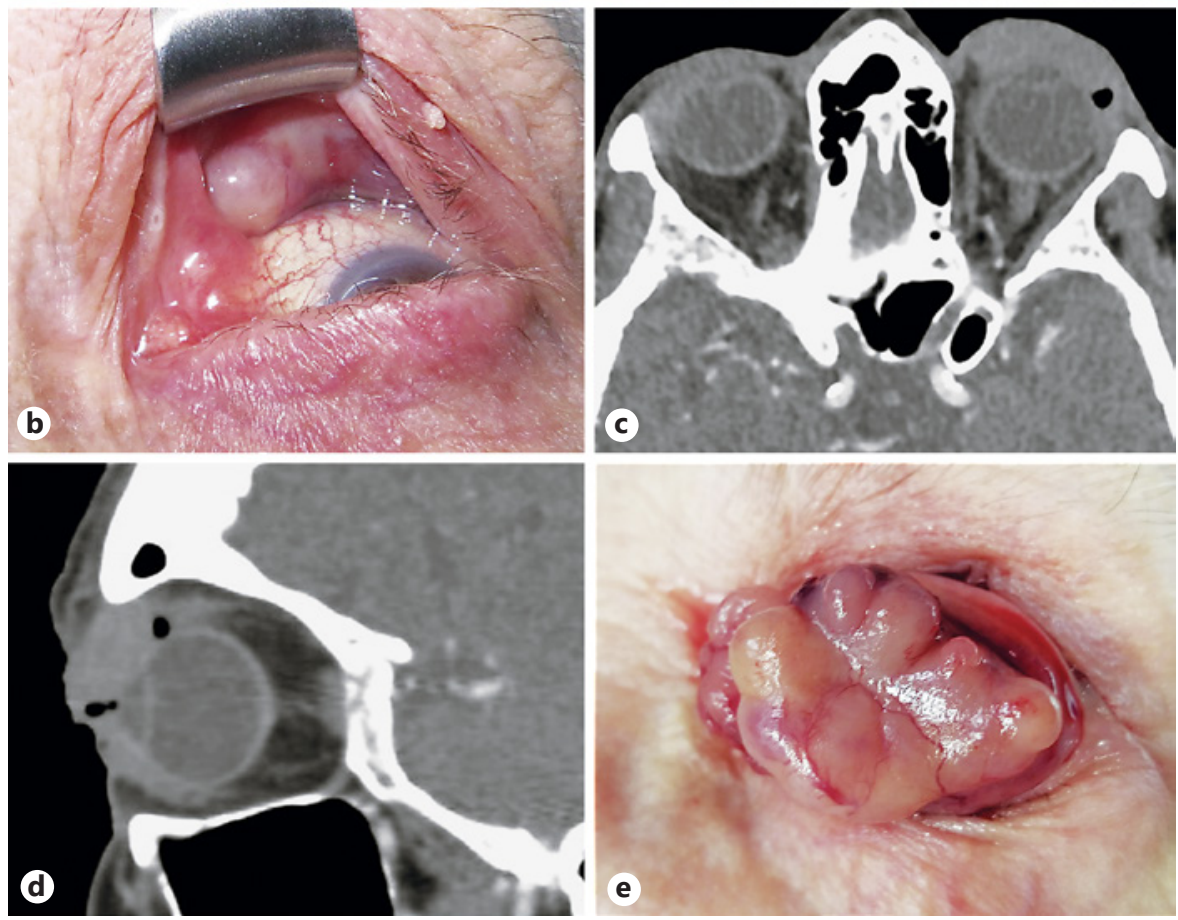

one can distinguish between disease of the anterior eyelid lamella (skin and orbicularis muscle) from that of the posterior lamella (tarsus and adherent palpebral conjunctiva). The tarsus is generally an effective barrier in preventing the spread of a conjunctival tumor into the skin and vice versa, but itself is virtually never the initial site of myeloblastic involvement. These important anatomic distinctions can be made by everting the eyelids, particularly the upper ones.

Myeloid sarcoma has many ophthalmic symptomatic manifestations [4-7]. Proptosis, lacrimal gland enlargement, orbital bone destruction, eyelid fullness, conjunctival masses, and anterior segment, choroidal and retinal infiltrates have all been observed. Proptosis accounts for $50-100 \%$ of the major signs [4-6], sometimes accompanied by ptosis and diplopia. Destructive bone foci are due to the preferential subperiosteal localization of the malig- nant deposits. Lesions surrounding the brain and parasellar region can produce neuro-ophthalmic symptoms and dysfunctions, but myeloid sarcomas of the brain parenchyma are vanishingly rare [8].

Fig. 2. Histopathologic and immunophenotypic characteristics of the tumor. Hypercellular, blastic round cell tumor displaying myriad mitoses. The cells are arranged in psedoepithelial groups. The inset depicts an abnormal megakaryoblast. The reticulin stain outlines the cellular clusters. Ki-67 positivity was $40 \%$ of the cells in the lesion. CD45 positivity, also known as leukocyte common antigen. CD43 positivity, a biomarker of granulocytes and their precursors. CD61 positivity, a biomarker of megakaryocytes. CD117 positivity, a biomarker of myeloid progenitor cells. a, b, inset $\mathrm{He}$ matoxylin and eosin, $\times 40(\mathbf{a}), \times 20(\mathbf{b}), \times 10$ (inset). c Reticulin, $\times 20$. $\mathbf{d}$-h Immunoperoxidase reaction, diaminobenzidine chromagen, hematoxylin counterstain, $\times 20, \times 40, \times 20, \times 40, \times 20$.

(For figure see next page.) 

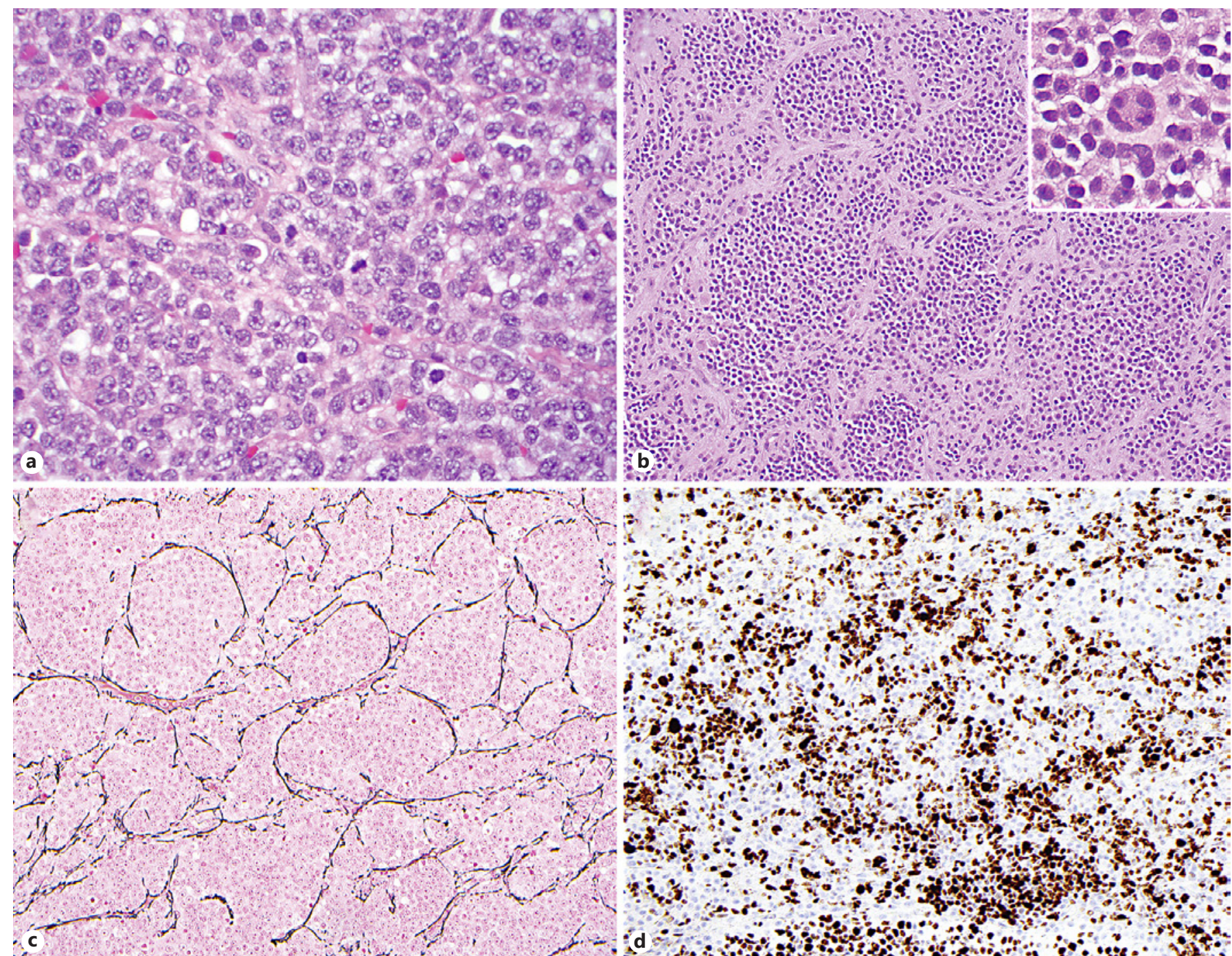

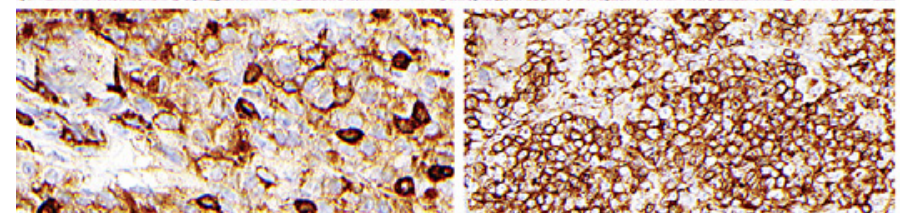

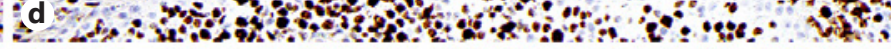

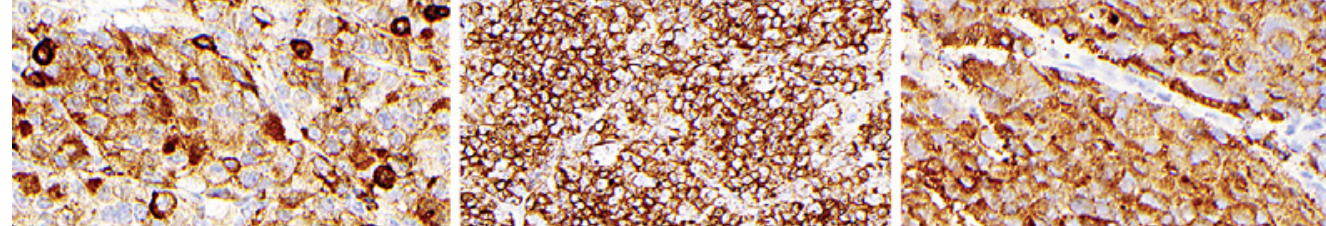

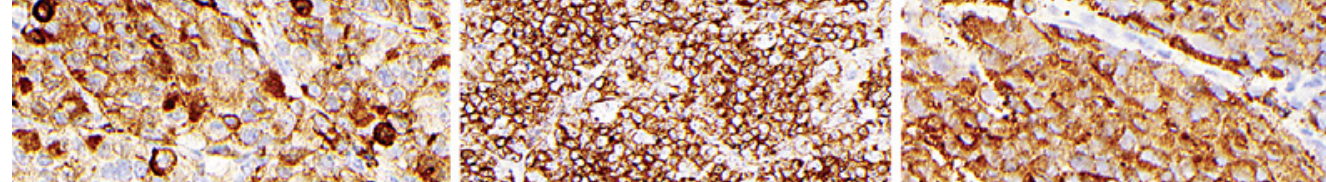

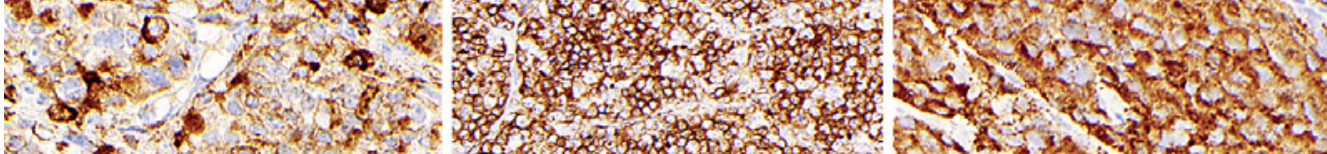

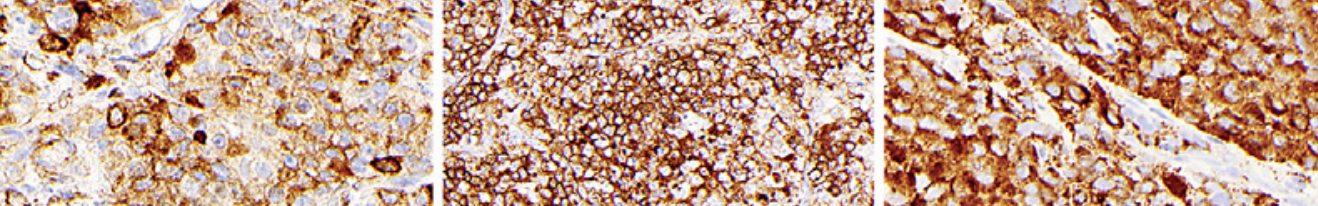

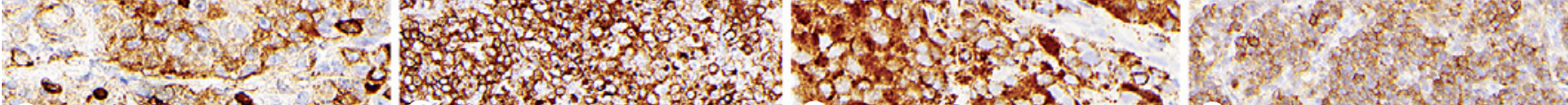

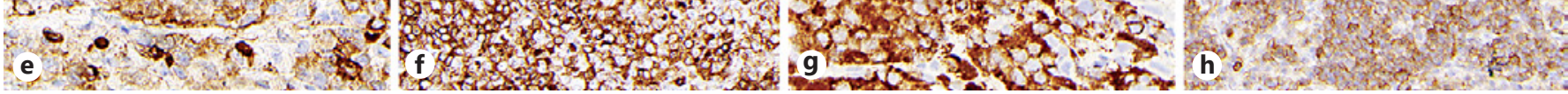


Three earlier reports of myeloid sarcoma of the conjunctiva exhibited unusual findings. A 90-year-old man with chronic myeloid leukemia developed unstoppable bloody tears (hemolacria) lasting at least several weeks and originating in a conjunctival myeloid sarcoma [9]. This case is different from most other conjunctival myeloid sarcomas in that it arose in a chronic myeloproliferation rather than in an acute one. Another conjunctival example was reported in an 8-year-old girl with bilateral epibulbar myeloid sarcomas [10]. A unique presentation occurred in a 34-year-old apparently healthy woman who displayed involvement of the palpebral conjunctiva of all 4 eyelids with red, papular lesions [11]. No evidence of leukemia was discovered at presentation after a systemic workup was performed; remarkably the patient was free of systemic disease after 15 months of follow-up. Skin disease of all 4 eyelids has also been reported; there is an analogous case of extranodal marginal zone lymphoma [12].

In 1975, a comprehensive report of granulocytic sarcoma (myeloid sarcoma) of the ocular adnexa was published that documented 33 cases in the files of the Armed Forces Institute of Pathology [5]. The authors determined, according to their cohort, that patients were more often children, with boys dominating over girls (Table 1 ). There was a propensity (two thirds of patients) for nonwhite ethnic groups (African, Pacific Rim) to be affected. Only 4 patients were already diagnosed with leukemia. To rule out a lymphoma or simulating round cell malignan$\mathrm{cy}$, the recommendation was made to employ the Leder esterase stain to identify eosinophilic metamyelocytes that facilitated the correct diagnosis. The prognosis for the patients was uniformly unfavorable, in that around two thirds died within a year from the onset of their ocular sarcomas. A patient not included in the AFIP series, however, developed leukemia 4 years after the appearance of the myeloid sarcoma [5]. To complete the spectrum of disease, there is another report of anterior segment intraocular infiltrates of myeloid sarcoma [13].

The subject of ocular adnexal myeloid sarcoma has also been reviewed in 3 other recent useful articles, but each individual case is presented with little detail. A study of 31 orbital cases of myeloid sarcoma assembled from a single institution (Prasad Eye Institute, Hyderabad, India) [6] generated some findings (Table 1) at variance with those from the AFIP. This disparity is likely the result of uncontrolled biases inherent in studies from a referral, consultative institute such as the AFIP. Table 1 lists the salient differences. In the Indian study, there was a higher percentage of concomitant leukemia, a slightly greater number of female patients, and an all Indian population. Patients with isolated myeloid sarcoma survived 43 months on average, and those who had leukemia 9.2 months. It was unclear whether or not the two groups had received therapy, but the presumption is that they did. A second smaller series of 10 cases originated from the M.D. Anderson Cancer Center [7]. There were 6 men and 4 women with a median age of 49 years (range 19-29 years); 6 had proptosis, 3 conjunctival lesions, and 1 retinal infiltrates. After apparently successful therapy for the ocular adnexal myeloid sarcoma, 4 patients subsequently developed another nonocular myeloid tumor in the skin and lung, breast, skin and lymph nodes, and central nervous system. In the third study, again from the Prasad Eye Institute [14], 12 patients with proptosis were evaluated. They had the following features: median age at presentation was 8.6 years (oldest 17 years), 7 females and 5 males, proptosis in all patients, a palpable orbital mass in $11 \mathrm{pa}$ tients, bilateral disease in 4 patients, subconjunctival hemorrhages in 1 patient, retinal or subhyaloid hemorrhages in 4 patients, and disc edema in 2 patients. A superior or superotemporal orbital location was common for the mass. The median duration of symptoms was 4 weeks (longest 20 weeks). The second Indian study probably incorporated the patients in the first, although this was not stated in the article. None of the previously published myeloid sarcoma cases in the eye literature has dealt with the diagnosis of acute megakaryoblastic leukemia, a form of myeloblastic proliferation, although 3 nonconjunctival ocular adnexal cases have been published in the nonophthalmic literature $[15,16]$.

Regarding the present case there was one distracting diagnosis - a possible vascular malignancy due to variable positivity for FLI-1, factor VIII, and ERG. However, further immunophenotyping led to the discovery of strongly positive staining for CD7, CD31, CD43, CD45, CD61, and $\mathrm{CD} 117$ in the bone marrow cells among the $8.8 \%$ myeloblasts that had the same morphology as those in the conjunctival lesion, supporting a lesion of myeloid lineage [1]. Furthermore, myeloperoxidase and lysozyme were focally positive. The dispersal of megakaryoblasts reinforced the impression of acute megakaryoblastic leukemia. Ki-67 positivity was approximately $40 \%$. Negative staining for biomarkers of nonhematopoietic round cell solid tumors (neuroblastoma, melanoma, and rhabdomyosarcoma) was unequivocally definitive.

To recapitulate: making the diagnosis of myeloid sarcoma depends on immunophenotyping that employs the most commonly expressed myeloid antigens/biomarkers [1]. These are, in descending order of frequency, CD68/ 
Table 1. Clinicopathological features of granulocytic sarcomas in two large studies ${ }^{\mathrm{a}}$

\begin{tabular}{lcc}
\hline & $\begin{array}{c}\text { Aggarwal } \\
\text { et al. [6] }\end{array}$ & $\begin{array}{c}\text { Zimmerman } \\
\text { and Font [5] }\end{array}$ \\
\hline Total cases & 31 & 33 \\
Sex, $n$ & & \\
Males & $11(36)$ & $19(58)$ \\
Females & $20(64)$ & $13(39)$ \\
Unspecified & 0 & $1(3)$ \\
Age & & \\
Range, years & $3-59$ & $1-60$ \\
Median, years & 12 & 7 \\
Children (<18 years), $n$ & $23(74)$ & $29(88)$ \\
$\quad$ Adults ( $\geq 18$ years), $n$ & $8(26)$ & $4(12)$ \\
Laterality, $n$ & & \\
Right & $13(42)$ & $1(3)$ \\
Left & $18(58)$ & $2(6)$ \\
$\quad$ Unspecified & 0 & $30(91)$ \\
Presenting symptoms, $n$ & & \\
Proptosis & $31(100)$ & $22(67)$ \\
Eyelid edema & $4(13)$ & $2(6)$ \\
Diminution of vision & $3(10)$ & $1(3)$ \\
Ptosis & $2(7)$ & $1(3)$ \\
Diplopia & $1(3)$ & unspecified \\
Association with hematological malignancy, $n$ & \\
Concomitant & $13(42)$ & $4(12)$ \\
Diagnosed during follow-up & $13(42)$ & $22(67)$ \\
Isolated granulocytic sarcoma ${ }^{b}$ & $5(16)$ & $5(15)$ \\
Unspecified & 0 & $3(10)$ \\
\hline
\end{tabular}

Figures in parentheses are percentages. ${ }^{\text {a }}$ Based on Table 1 in Aggarwal et al. [6] and Table 1 in Zimmerman and Font [5]. ${ }^{\mathrm{b}}$ Lesions not yet established to be associated with leukemia.

KPI, MPO (myeloperoxidase), CD117, CD99, CD68/PGM1, lysozyme, CD34, TdT, CD56, CD61/LAT/von Willebrand antigen, CD30, glycophorin, and CD4. T-cell markers may anomalously be expressed. Another underutilized common genetic biomarker for detecting myeloid sarcoma is cytoplasmic mutated nucleophosmin (NPM) reflective of NPM1 mutation [17]. The foregoing biomarkers need to be applied judiciously for making a satisfactory diagnosis. CD42, normally encountered on mature megakaryocytes, may be found on a subset of tumor cells. With regard to megakaryocytes a panel of probes is available, and with them the level of maturation can determine expression. Morphologically megakaryocytes have ample cytoplasm and a low nuclear to cytoplasmic ratio, while megakaryoblasts evince the opposite. In this regard, CD41b, CD42b, CD61, von Willebrand factor, and LAT (linker for activation of T cells) can be high- ly revealing of the presence of megakaryocytes. Unfortunately, not all of these biomarkers were available to us in our pathology laboratories.

The main chromosomal abnormalities detected in the current case were the presence of two clones: trisomy 8 and a $\mathrm{t}(12 ; 17)$ translocation. Trisomy 8 in an elderly patient tends to worsen the prognosis [3]. Documented genetic abnormalities in other cases are trisomy and tetrasomy 21 (trisomy 21 also causes Down's syndrome with its enhanced tendency to develop acute lymphocytic or myelogenous leukemia - overall risk 2-3\%), monosomy 7, MLL rearrangement, INV(16), trisomy 4, monosomy 16, trisomy 11, NEMI mutation, and in pediatric (but rarely older) patients, a $t(8: 21)$ translocation [18]. Furthermore, a bone marrow biopsy of our patient disclosed increased amounts of reticulin fibers and an abnormally hypercellular marrow comprised of 8-9\% myeloblasts, with multilineage dysplasia of erythrocytes (poikilocytosis, binucleation, nuclear budding) and megakaryocytes (abnormal colony formation), and abnormal myelopoiesis with a left shift displaying hypogranularity and aberrant segmentation of the myeloblasts. The megakaryocytic elements were increased in number, interpreted as blastoid (having single and multiple nucleoli) that displayed a scattered arrangement in large clumps.

Over the patient's clinical course, two syndromes were diagnostically considered: a myelodysplastic syndrome and primary myelofibrosis. The myelodysplastic syndromes [19-22] constitute a complex cluster of precursor marrow states. These conditions eventually evolve into full-blown leukemias deriving from altered clonal stem cells. A peripheral blood cytopenia often coexists paradoxically with a hypercellular bone marrow containing sheets of large atypical cells. All marrow cell lineages are affected. The inciting mutation for the syndrome is yet to be determined but it is now known that leukemic transformation implicates genes in the RAS pathway [19]. Recent genetic findings implicate mutations in TET2 and ASXL1 [20, 23] along with GATA2, SF3DS, TERT, and TERC. An unanswered question is whether primary myelofibrosis [24-27] should be subsumed under the rubric of myelodysplastic syndromes. In myelofibrosis the marrow hematopoietic cells are ultimately replaced with collagen produced by fibroplasia. It is likely that the myelodysplastic syndrome encompasses disparate and molecularly distinct entities possibly embracing myelofibrosis which culminates in a fibrotic state concomitant with myeloproliferation. Our patient had increased deposition of reticulin fibers in her bone marrow biopsy that could be interpreted as suggestive of early myelofibrosis. 
Primary myelofibrosis is a rare, enigmatic bone marrow hematopoietic neoplasm [24-28]. It is also termed agnogenic myeloid metaplasia and can be responsible as a prodromal state for the emergence of multilineal stem cell disease, accompanied by progressive fibrosis of the marrow cavities. There is displacement of hematopoiesis to alternative extramedullary sites (liver, spleen, lymph nodes). Most lesions are idiopathic, but the disease may be associated with polycythemia vera or essential thrombocythemia. Jak2, CALR, MPL and V617F gene mutations play a central role in the genesis of the disease [23, 25]: V671F makes stem cells more sensitive to growth factors that require JAK2 for signal transduction [29]. Another gene, MPL, serves as a receptor for thrombopoietin. The W515 mutation leads to the overproduction of abnormal megakaryocytes, as seen in the present patient, by means of proliferation of an errant thrombopoietic receptor protein. The megakaryocytes then stimulate fibroblasts to produce collagen in the bone marrow compartment.

While there are overlaps between myelofibrosis and myelodysplasia, there are also significant clinical and genetic differences leaving our understanding fragmentary and incomplete. In the present case, these issues led to some perplexity on the part of clinicians managing the current case, with the best diagnosis ultimately rendered by the hematopathology consultants being myelodysplastic leukemia and sarcoma. It is agreed, however, that both conditions are monoclonal and preleukemic. A granulocytic sarcoma originates more often in the setting of myelodysplasia than primary myelofibrosis.

The major differential diagnoses for myeloid sarcoma with megakaryocytic/blastic differentiation are lymphoma and malignant soft tissue round cell tumors. Lymphoma (apart from Burkitt lymphoma with its distinctive starry sky appearance) is utterly rare in childhood but does occur in our patient's age group (87 years old). Orbital bone destruction is comparatively uncommon in lymphoma. Blastic hematopoietic cells have round or slightly reniform nuclei with a finely stippled heterochromic pattern ("open chromatin"), prominent nucleoli, and fine cytoplasmic granules in scattered cells. Lymphoma cells, on the other hand, have coarser heterochromatin with a tendency toward perinucleolar clearing or halo formation. Except for large B cell lymphoma, the Ki-67 proliferation index for smaller cell lymphomas is lower than that of hematoblastic neoplasms. Also, myeloid sarcoma is more likely to involve the subperiosteal space causing bone changes. The B cell markers will be different in ocular adnexal lymphoma (most are of B cell lineage), and there is an absence of lysozyme, muramidase, and cytoplasmic NPM expression [17].

Simulating solid soft tissue round cell tumors can be separated from myeloid sarcomas by their CD43-negative immunostaining and from lymphoma by the absence of $\mathrm{T}$ and $\mathrm{B}$ cell biomarkers. Particularly rhabdomyosarcoma (desmin and myogenin positive) and metastatic neuroblastoma (osteolytic and NB84, MAP-2, neurofilament, synaptophysin, protein gene product $9.9, \beta$-catenin, CD56 and neuron-specific enolase positive) $[30,31]$ must be eliminated; Ewing's sarcoma/primitive neuroectodermal tumor expresses NKX2.2, CD99, and FLI1 [32, 33], while neuroendocrine tumors are best identified with synaptophysin and chromogranin immunostaining [34, 35]. Epithelioid melanoma cells can be excluded by S100, MART-1 and Sox10 negativity. Polygonal epithelial cells are ruled out by negativity of various cytokeratins (especially pan-cytokeratin) and of p63, as well as by vimentin positivity.

While it is not the focus of this article, a few comments are offered about therapy [36]. The mainstay is chemotherapy. Most reports have consisted of small series with various chemotherapeutic regimens. The role of radiotherapy is limited but can probably be used for local lesions such as periocular myeloid sarcomas or those involving vital organs. Stem cell transplantation is employed when there appears to have been near eradication of the leukemic process by other modalities.

\section{Acknowledgments}

The authors express their gratitude for the consultations of Drs. Robert P. Hasserjian and Judith A. Ferry of the Hematopathology Division of the Pathology Department at the Massachusetts General Hospital, who guided the authors toward the correct diagnosis.

Natalie Wolkow is supported by a Heed Ophthalmic Fellowship.

\section{Statement of Ethics}

The study followed the tenets of the Declaration of Helsinki and was approved by the Massachusetts Eye and Ear Infirmary Institutional Review Board.

\section{Disclosure Statement}

The authors declare no conflicts of interest.
Jakobiec/Wolkow/Zakka/Rubin 


\section{References}

1 Pileri S, Orazi A, Fallini B: Myeloid sarcoma; in Swerdlow S, Campo E, Harris N, Jaffe E, Pileri S, Stein H, Thiele J (eds): WHO Classification of Tumours of Haematopoietic and Lymphoid tissues. Lyon, International Agency for Research on Cancer, 2017, pp 167-168.

2 Arber DA: Myeloid sarcoma; in Jaffe ES, Arber DA, Campo E, Harris NL, QuintanillaMartinez L (eds): Hematopathology. Philadelphia, Elsevier, 2017, pp 842-845.

- 3 Koh Y, Kim I, Bae JY, Song EY, Kim HK, Yoon SS, Lee DS, Park SS, Park MH, Park S, Kim BK: Prognosis of secondary acute myeloid leukemia is affected by the type of the preceding hematologic disorders and the presence of trisomy 8. Jpn J Clin Oncol 2010; 40:1037-1045.

4 Sharma T, Grewal J, Gupta S, Murray PI: Ophthalmic manifestations of acute leukaemias: the ophthalmologist's role. Eye (Lond) 2004; 18:663-672.

5 Zimmerman LE, Font RL: Ophthalmologic manifestations of granulocytic sarcoma (myeloid sarcoma or chloroma). The third Pan American Association of Ophthalmology and American Journal of Ophthalmology Lecture. Am J Ophthalmol 1975;80:975-990.

-6 Aggarwal E, Mulay K, Honavar SG: Orbital extra-medullary granulocytic sarcoma: clinicopathologic correlation with immunohistochemical features. Surv Ophthalmol 2014;59: 232-235.

7 Ohanian M, Pemmaraju N, Rozovski U, Alat$\operatorname{tar}$ ML, Estrov Z, Kundra V, Tung C, Ravandi F, Manning J, Abruzzo LV: Ocular extramedullary myeloid leukaemia. Br J Haematol 2018;180:738-740.

-8 Novello M, Coli A, Della Pepa GM, Martini M, Doglietto F, De Stefano V, Bellesi S, Pescarmona E, Lauriola L: Myeloid sarcoma with megakaryoblastic differentiation mimicking a sellar tumor. Neuropathology 2014;34:179184.

9 Shah SB, Reichstein DA, Lally SE, Shields CL: Persistent bloody tears as the initial manifestation of conjunctival chloroma associated with chronic myelogenous leukemia. Graefes Arch Clin Exp Ophthalmol 2013;251:991992.

$\checkmark 10$ Rosenberg C, Finger PT, Furlan L, Iacob CE: Bilateral epibulbar granulocytic sarcomas: a case of an 8-year-old girl with acute myeloid leukaemia. Graefes Arch Clin Exp Ophthalmol 2007;245:170-172.

-11 Thomas SA, Durairaj VD: Isolated myeloid sarcoma presenting in all four eyelids. Ophthal Plast Reconstr Surg 2007;23:336-338.
12 Nguyen HV, Jakobiec FA, Zakka FR, Yoon MK: Bilateral upper and lower eyelid margin swelling and madarosis due to lymphoma. Surv Ophthalmol 2017, Epub ahead of print.

13 Dunbar KE, Hubbard GB 3rd, Wells JR: Anterior chamber infiltrate in 3-month-old: rare presentation of myeloid sarcoma. Eye (Lond) 2012;26:1274-1275.

14 Murthy R, Vemuganti GK, Honavar SG, Naik M, Reddy V: Extramedullary leukemia in children presenting with proptosis. J Hematol Oncol 2009;2:4

15 Slavc I, Urban C, Haas OA, Kroisel PM, Koller $\mathrm{U}$ : Acute megakaryocytic leukemia in children. Clinical, immunologic, and cytogenetic findings in two patients. Cancer 1991;68: 2266-2272.

16 Cetin N, Lorsbach RB: Pan myeloid antigennegative pediatric acute megakaryoblastic leukemia. Pediatr Blood Cancer 2014;61: 2089-2091.

17 Falini B, Lenze D, Hasserjian R, Coupland S, Jaehne D, Soupir C, Liso A, Martelli MP, Bolli N, Bacci F, Pettirossi V, Santucci A, Martelli MF, Pileri S, Stein H: Cytoplasmic mutated nucleophosmin (NPM) defines the molecular status of a significant fraction of myeloid sarcomas. Leukemia 2007;21:1566-1570.

18 Schwyzer R, Sherman GG, Cohn RJ, Poole JE, Willem P: Granulocytic sarcoma in children with acute myeloblastic leukemia and $\mathrm{t}(8 ; 21)$. Med Pediatr Oncol 1998;31:144-149.

19 Lindsley RC: Uncoding the genetic heterogeneity of myelodysplastic syndrome. Hematology Am Soc Hematol Educ Program 2017; 2017:447-452.

20 Tefferi A, Vardiman JW: Myelodysplastic syndromes. N Engl J Med 2009;361:1872-1885.

21 Hasserjian R, Head D: Myelodysplastic syndromes; in Jaffe ES, Arber DA, Campo E, Harris NL, Quintanilla-Martinez L (eds): Hematopathology. Philadelphia, Elsevier, 2017, pp 793-815.

22 Hyjek E, Vardiman JW: Myelodysplastic/myeloproliferative neoplasms; in Jaffe ES, Arber DA, Campo E, Harris NL, Quintanilla-Martinez L (eds): Hematopathology. Philadelphia, Elsevier, 2017, pp 883-910.

23 Tefferi A: Novel mutations and their functional and clinical relevance in myeloproliferative neoplasms: JAK2, MPL, TET2, ASXL1, CBL, IDH and IKZF1. Leukemia 2010;24: $1128-1138$

24 Thiele J, Kvasnicka H, Orazi A, Gianelli U, Barbo T, Barcsi G, Tefferi A: Primary myelofibrosis; in Swerdlow S, Campo E, Harris N, Jaffe E, Pileri S, Stein H, Thiele J (eds): WHO Classification of Tumours of Haematopoietic and Lymphoid Tissues. Lyon, International Agency for Research on Cancer, 2017, pp 44-50.
25 Tefferi A: Clinical manifestations and diagnosis of primary myelofibrosis; in Schrier S, Rosmarin A (eds): UpToDate. Waltham, Wolters Kluwer, 2017.

26 Lichtman MA: Is it chronic idiopathic myelofibrosis, myelofibrosis with myeloid metaplasia, chronic megakaryocytic-granulocytic myelosis, or chronic megakaryocytic leukemia? Further thoughts on the nosology of the clonal myeloid disorders. Leukemia 2005;19: 1139-1141.

27 Tefferi A: Primary myelofibrosis: 2014 update on diagnosis, risk-stratification, and management. Am J Hematol 2014;89:915-925.

28 Vardiman JW: Primary Myelofibrosis; in Jaffe ES, Arber DA, Campo E, Harris NL, Quintanilla-Martinez L (eds): Hematopathology. Philadelphia, Elsevier, 2017, pp 869-876.

29 Staerk J, Constantinescu SN: The JAK-STAT pathway and hematopoietic stem cells from the JAK2 V617F perspective. JAKSTAT 2012; 1:184-190.

30 Shin S, Treaba D, Delellis R: Neuroblastoma; in Dabbs DJ (ed): Diagnostic immunohistochemistry: theranostic and genomic applications. Philadelphia, Elsevier/Saunders, 2014, pp 349-351.

31 Zhu S, Markku M, Lin G: Markers for neuroblastoma; in Lin F, Prichard J (eds): Handbook of Practical Immunohistochemistry: Frequently Asked Questions. New York, Springer, 2015, p 575.

32 Coffin C, Cajaiba M, Lyle P, Correa H, Black $\mathrm{J}$ : Ewing sarcoma/primitive neuroectodermal tumor; in Dabbs DJ (ed): Diagnostic Immunohistochemistry: Theranostic and Genomic Applications. Philadelphia, Elsevier/Saunders, 2014, pp 862-865.

33 Zhu S, Miettinen M, Lin G: Markers for Ewing sarcoma and primitive neuroectodermal tumor (ES/PNET); in Lin F, Prichard J (eds): Handbook of Practical Immunohistochemistry: Frequently Asked Questions. New York, Springer, 2015, p 578.

34 Bhargava R, Dabbs DJ: Neuroendocrine antibodies; in Dabbs DJ (ed): Diagnostic Immunohistochemistry: Theranostic and Genomic Applications. Philadelphia, Elsevier/Saunders, 2014, pp 224-225.

35 MacIntosh PW, Jakobiec FA, Stagner AM, Gilani S, Fay A: High grade neuroendocrine neoplasm of the antrum and orbit. Surv Ophthalmol 2015;60:486-494.

36 Avni B, Koren-Michowitz M: Myeloid sarcoma: current approach and therapeutic options. Ther Adv Hematol 2011;2:309-316. 